\title{
Tenosynovitis caused by Scedosporium apiospermum infection misdiagnosed as an Alternaria species: a case report
}

\author{
Choon-Mee Kim ${ }^{1 \dagger}$, Sung-Chul Lim² ${ }^{2 \dagger}$, Joa Kim³ ${ }^{3}$ Hoe-Soo Jang ${ }^{3}$, Jong-Hun Chung ${ }^{3+}$, Na-Ra Yun ${ }^{3}$, Dong-Min Kim ${ }^{3^{*} \dagger}$,
} Piyush Jha ${ }^{3}$, Babita Jha ${ }^{3}$, Seok Won $\mathrm{Kim}^{4}$, Sook Jin Jang ${ }^{5}$ and Jong Hee Shin ${ }^{6}$

\begin{abstract}
Background: Scedosporium apiospermum, which can usually be isolated from soil, polluted stream water and decaying vegetation, is increasingly recognized as an opportunistic dematiaceous fungus. The mortality rate of infection in immunocompromised hosts is over $50 \%$. S. apiospermum is commonly responsible for dermal and epidermal infections (i.e., mycetoma) after traumatic penetration.

Case presentation: A 73-year-old woman was admitted to our hospital complaining of painful swelling and tenderness on the dorsum of the proximal left wrist and hand. The symptoms had persisted for approximately 2 months. A physical examination revealed a $4 \times 3 \mathrm{~cm}$, poorly defined, erythematous papule, which was fluctuant, with pustules and crusts on the dorsum of the left hand.

Conclusions: We report a very rare case of tenosynovitis caused by S. apiospermum infection. We identified the infectious agent via molecular DNA sequencing. The infectious agent was initially misidentified as an Alternaria species by microscopic examination with lactophenol cotton blue (LPCB) staining. The infection was successfully treated with debridement and adjuvant fluconazole therapy.
\end{abstract}

Keywords: Scedospermum apiospermum, Tenosynovitis, Debridement, Case report

\section{Background}

Scedosporium apiospermum is an asexual anamorph of the fungus Pseudallescheria boydii and can usually be isolated from soil, polluted stream water and decaying vegetation [1]. This pathogen accounts for approximately $20 \%$ of all non-Aspergillus mold infections in organ transplant recipients [2]. S. apiospermum is increasingly recognized as an opportunistic fungus. The infection mortality rate is over $50 \%$ in immunocompromised hosts [3, 4]. Here, we report a case of tenosynovitis caused by an S. apiospermum infection that was initially misidentified as an Alternaria species. The infection was successfully treated with debridement and adjuvant fluconazole therapy.

\footnotetext{
* Correspondence: drongkim@chosun.ac.kr

${ }^{\dagger}$ Equal contributors

Choon-Mee Kim and Sung-Chul Lim contributed equally to this work. Jong-Hun Chung and Dong-Min Kim contributed equally to this work. ${ }^{3}$ Department of Internal Medicine, School of Medicine, Chosun University, Gwangju, Republic of Korea

Full list of author information is available at the end of the article
}

\section{Case presentation}

A 73-year-old woman was admitted to Chosun University Hospital, Gwang-ju, Korea. She was complaining of painful swelling and tenderness on the dorsum of the proximal left wrist and hand. The symptoms had persisted for approximately 2 months. A local clinic suspected cellulitis and administered cefoperazone/sulbactam for 10 days. However, the wound became worse, and erythema formed on the left hand. She had a clinical history of hypertension (20 years), diabetes ( 2 months), and a left distal radial and ulnar styloid process fracture that had occurred 2 years prior. In addition, she had undergone a discectomy at the L4-5 level one year previously.

A physical examination revealed a $4 \times 3 \mathrm{~cm}$, poorly defined, erythematous papule which was fluctuant, with pustules and crusts on the dorsum of left hand. Movements of the wrist, hand and fingers were slightly restricted and painful. Aspirated fluid from the lesion was obtained for mycological examination, and skin 
biopsies were obtained for histological and mycological examination.

The laboratory findings revealed that the white blood cell (WBC) count was $9360 / \mathrm{mm}^{3}$, the erythrocyte sedimentation rate (ESR) was $24 \mathrm{~mm} / \mathrm{h}$, and the C-reactive protein (C-RP) level was $1.13 \mathrm{mg} / \mathrm{dL}$. An X-ray of the left hand revealed a healed fracture of the distal radius. Magnetic resonance imaging (MRI) of the left wrist revealed cellulitis of the dorsum of the wrist and hand and tenosynovitis of the extensor tendons (Fig. 1). Histological sections of the skin biopsies were stained with Gomori methenamine silver and revealed acute and chronic granulomatous inflammation with fungal hyphae and spores.

The clinical specimens were cultured on Sabouraud's dextrose agar (SDA) containing chloramphenicol at $30{ }^{\circ} \mathrm{C}$. On the 9th day, the culture yielded grey soft fungus on the front and dark-brown fungus on the other side. The
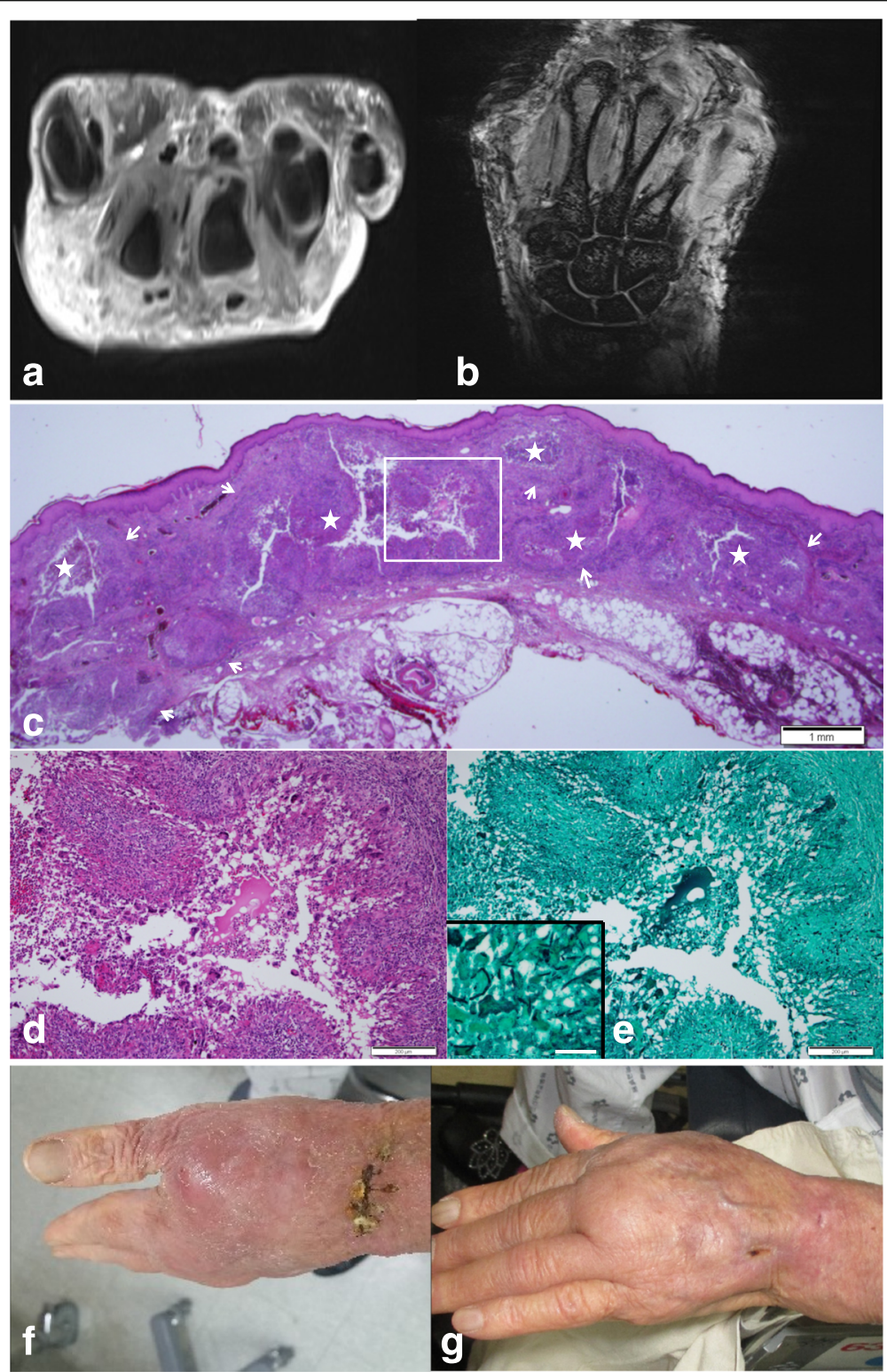

Fig. 1 a MRI T2 weighted contrast transverse image reveals diffuse edematous swelling in the dorsum of the wrist, fluid in the extensor tendon sheath and fluid collection in the distal radio-ulnar joint. b MRI T1 weighted coronal image reveals diffuse paratendinous fluid in the extensor tendon sheath. c Skin biopsy shows multiple variable-sized granulomas (arrows) bearing central microabscesses (asterisks) in the dermis. H\&E staining, Scale bar measures $1 \mathrm{~mm}$. $\mathbf{d}$ Higher magnification of the image; a boxed area shows a well-formed palisading granuloma consisting of epithelioid cells, lymphocytes and multinucleated giant cells. H\&E staining, Scale bar measures $200 \mu \mathrm{m}$. e Silver staining demonstrates septated fungal hyphae in the granuloma. Gomori methenamine silver staining, Scale bar measures $200 \mu \mathrm{m}$. Inset shows a higher magnification of the fungal hyphae. Scale bar measures $50 \mu \mathrm{m}$. f Skin photo on the dorsum of the left hand (before treatment). $\mathbf{g}$ Skin photo on the dorsum of the left hand (after treatment one month later) 
clinical isolate was initially identified as an Alternaria species via a microscopic examination with lactophenol cotton blue (LPCB) staining. No other microorganisms were isolated from the clinical specimens. Because the wounds had improved, we continued treatment with wound irrigation, debridement and adjuvant fluconazole therapy with IV fluconazole $400 \mathrm{mg}$ once a day for 14 days and changed to oral fluconazole $200 \mathrm{mg}$ for 24 days. DNA sequencing of total genomic DNA extracted from purified colonies was performed to confirm the microbiological species.

Gene sequence analysis of the ITS region and D1/D2 regions of the $26 \mathrm{~S}$ ribosomal DNA of rRNA genes was carried out. The ITS region (including the 5.8S rRNA gene) and 26S rRNA gene D1/D2 domains were amplified with the primer pair pITS-F (5'-GTCGTAACAAGGTTAACC TGCGG-3') and pITS-R (5'-TCCTCCGCTTATTGATAT GC- $3^{\prime}$ ) and the primer pair NL1 (5'-GCATATCAATAAG CGGAGGAAAAG-3') and NL4 (5'-GGTCCGTGTTTCA AGACGG-3'), respectively [5]. The fungus was identified (www.ncbi.nlm.nih.gov/BLAST) as Pseudallescheria boydii (anamorph: S. apiospermum) with 100\% homology according to the BLASTn program.

In vitro susceptibility testing of the mold isolate against amphotericin B, itraconazole, posaconazole, voriconazole, caspofungin and micafungin was performed by a broth microdilution assay according to the methods of the Clinical and Laboratory Standards Institute (CLSI) M38A2 [6]. Briefly, $100 \mu$ l culture preparations (standard RPMI 1640 broth [0.2\% dextrose], final inoculum concentrations ranging from $0.4 \times 10^{4}$ to $5 \times 10^{4} \mathrm{CFU} / \mathrm{ml}$ ) were inoculated into 96-well microtiter plates containing $100 \mu \mathrm{l}$ drug dilutions, and the plates were incubated at $35^{\circ} \mathrm{C}$ for $48 \mathrm{~h}$. The minimum inhibitory concentrations (MICs) for amphotericin B, itraconazole, posaconazole, and voriconazole were determined visually as the lowest concentrations with no visible growth (100\% inhibition) [6]. For echinocandins (caspofungin and micafungin), the end points were determined as the lowest concentrations of drug leading to the growth of small, rounded, compact hyphal forms in comparison to the hyphal growth observed in the control well (MEC, minimal effective concentration). The quality control strains Candida krusei ATCC 6258 and C. parapsilosis ATCC 22019 were employed as controls.

A follow-up culture of the wound was performed after 1 month and revealed no fungal growth. Ultimately, we reached a definitive diagnosis of tenosynovitis caused by S. apiospermum.

The patient was treated with oral fluconazole $200 \mathrm{mg}$ for 4 weeks. After her discharge from the hospital, we obtained the results of in vitro susceptibility testing of the isolates obtained from the aspirated fluid (Table 1). The last follow-up in the outpatient clinic was 4 months
Table 1 The antifungal susceptibility tests

\begin{tabular}{lll}
\hline Antifungal & \multicolumn{2}{l}{$100 \%$ inhibition MIC $(\mu \mathrm{g} / \mathrm{ml})$} \\
\cline { 2 - 3 } & $24 \mathrm{~h}$ & $48 \mathrm{~h}$ \\
\hline Itraconazole & 4 & $>16$ \\
Voriconazole & 1 & 1 \\
Posaconazole & 2 & $>16$ \\
Fluconazole & 1 & $>64$ \\
Amphotericin B & 1 & 4 \\
Micafungin & - & $4(\mathrm{MEC})$ \\
Caspofungin & - & 4 (MEC)
\end{tabular}

Cf $>M E C$ minimal effective concentration

after treatment. The patient recovered completely, with no recurrence.

\section{Discussion}

We report a case of tenosynovitis caused by S. apiospermum, which was identified via molecular DNA sequencing. The infectious agent was initially misidentified as an Alternaria species via a microscopic examination with lactophenol cotton blue staining. The dematiaceous fungi are defined by melanin or melanin-like pigmentation in the walls of the hyphae and/or spores.

Dematiaceous fungi (melanized fungi), including the Alternaria and Scedosporium species, cause a wide range of diseases from dermal and epidermal infections in immunocompetent hosts to disseminated sepsis in immunosuppressed hosts [7]. Alternaria species can cause human diseases such as cutaneous and subcutaneous infections, sinusitis, ocular infections and, on rare occasions, granulomatous lung disease (especially in immunocompromised hosts). The main clinical manifestation is allergenic respiratory symptoms, which are due to the inhalation of spores. Skin and cutaneous alternariosis mainly occur in immunocompromised hosts [8]. Alternaria species are commonly considered to be contaminants. Alternaria infections remain locally invasive without any dissemination in most cases. Nonetheless, infections caused by these organisms can be localized, extend to the surrounding tissues (deep extension), or disseminate (hematogenously) to distant organs. Therefore, Alternaria dissemination to other tissues should be histologically verified [9]. Fatal Alternaria infections were seldomly reported until recently.

However, Scedosporium species cause a broad spectrum of clinical diseases such as bronchopulmonary tree infections, localized cutaneous and subcutaneous infections, deep extension or dissemination to distal organs, septic arthritis, osteomyelitis, pneumonia, endocarditis, meningitis and brain abscess [10]. Scedosporium has been increasingly reported as a cause of severe infections in immunocompromised hosts; it is known to be pathogenic to both immunocompetent and immunocompromised hosts. Infections due to Scedosporium species can result in more than 
$50 \%$ mortality in immunocompromised hosts $[3,4]$. Thus, the exact identification of the pathogen is vitally important. The clinical isolate was initially identified as an Alternaria species upon a microscopic examination with lactophenol cotton blue staining. However, molecular DNA sequencing revealed that the infection was due to $S$. apiospermum. Tenosynovitis caused by S. apiospermum is very rare. Only one case of tenosynovitis (after a dog bite) has been reported world-wide [11]. Previously published data suggest that skin infections due to dematiaceous fungi may be more common in hosts whose activities carry a risk of penetrating trauma. Our patient did not report any recent trauma but lives in a rural area and performed agricultural activities. The definitive diagnosis of a fungal infection relies on the results of clinical mycological cultures and histological findings [12]. One of the studies by Sangoi et al. [13] revealed that fungal pathogens are misclassified in approximately $21 \%$ of patients with fungal infections diagnosed histologically but failed to show filamentous fungi when compared with cultures. The histological findings in our case revealed acute and chronic granulomatous inflammation with fungal hyphae and spores, which were stained with Gomori methenamine silver, and the clinical cultures indicated an Alternaria species. Through further investigation with DNA sequencing, the infectious agents were identified as Scedosporium apiospermum and Pseudallescheria boydii.

There is no consensus on the appropriate treatment of dematiaceous fungi infections [14]. Surgical intervention is an important element in the successful treatment of dermal and epidermal infections [15]. In our patient, the treatment consisted of debridement and adjuvant fluconazole therapy based on the mycological culture finding of an Alternaria species. There are a few published reports describing successful outcomes with voriconazole for the treatment of S. apiospermum in immunosuppressed hosts [16]. Lackner et al. [17] reported low MIC values for voriconazole (MIC 90 with $\leq 2 \mathrm{ug} / \mathrm{ml}$ ) in treating S. apiospermum. The MIC distribution of other antifungal agents, such as azole and echinocandin, did not reveal any appropriate activity against Scedosporium species. The in-vitro susceptibility test with the clinical isolates of our patient showed resistance to fluconazole, itraconazole and posaconazole after $48 \mathrm{~h}$. The infection was susceptible to other antifungals such as voriconazole, amphotericin B, micafungin, and caspofungin. MIC or MEC breakpoints have not been established for $S$. apispermum testing. Therefore, the clinical relevance of testing remains uncertain, and breakpoints with proven relevance have yet to be identified or approved by CLSI or any regulatory agency. As an MIC below $1 \mu \mathrm{g} / \mathrm{ml}$ is usually reported for most molds, including Aspergillus species, isolates were grouped as susceptible (MIC or MEC $\leq 1 \mu \mathrm{g} / \mathrm{ml}$ ), intermediate (MIC or MEC $2 \mu \mathrm{g} / \mathrm{ml}$ ) or resistant (MIC or MEC $4 \geq \mu \mathrm{g} / \mathrm{ml}$ ) for all drugs (CLSI M38). The in vitro susceptibility data are consistent with previous reports. Of the four antifungals tested in this study, voriconazole was found to be the most active against Scedosporium species, whereas other antifungals showed limited activity. Skin infections caused by dematiaceous fungi, including Alternaria species and S. apiospermum, are treated mainly with surgical interventions. Alternaria species are susceptible to adjuvant anti-fungal agents, such as fluconazole, but Scedosporium species have multidrug-resistant characteristics against many antifungal agents, including azoles and echinocandins [18]. Disseminated infections of Scedosporium species. should be treated with antifungal agents. Thus, it is important to distinguish Alternaria species from S. apiospermum.

In our case with a localized dermal and epidermal infection due to $S$. apiospermum in an immunocompetent patient, irrigation and debridement were more important than anti-fungal treatment.

\section{Conclusions}

This report presents the first case of S. apiospermum identified by molecular DNA sequencing, which was initially misidentified as an Alternaria species. Physicians should be aware that even though the culture results may indicate an Alternaria species infection upon a microscopic examination with lactophenol cotton blue staining, an S. apiospermum infection may also be present.

\section{Abbreviations \\ C-RP: C-reactive protein; ESR: Erythrocyte sedimentation rate; ITS: Internal transcribed spacer; LPCB: Lactophenol cotton blue; MRI: Magnetic resonance imaging; rDNA: Ribosomal DNA; SDA: Sabouraud's dextrose agar; WBC: White} blood cell

\section{Acknowledgements}

The authors are grateful to the patient for full consent to the publication of this case report.

Funding

This study was supported by research funds from Chosun University, 2014.

Availability of data and materials

The datasets supporting the conclusions of this article are included within additional files.

\section{Authors' contributions}

DMK designed and coordinated the study and contributed to drafting and reviewing the manuscript during the course of submission. CMK collected the patient's clinical data, wrote the manuscript, and revised the draft during the course of submission. SCL collected the patient's clinical data, pathologic data, and revised the draft during all over the course of resubmission. JHC were directly responsible for the patient and performed the clinical examinations and contributed to drafting and reviewing the manuscript during the course of submission. JK, and HSJ were directly responsible for the patient and performed the clinical examinations. NRY, SWK, and JHS supervised the clinical case interpretation and revised the manuscript. PJ carried out the molecular analysis. BJ and SJJ cultured the clinical specimens, performed microscopic examinations and helped to draft the manuscript. All the authors read and approved the final version of the manuscript.

Competing interests

The authors declare that they have no competing interests. 


\section{Consent for publication}

Signed informed consent was obtained from the patient for the publication of this case report.

\section{Ethics approval and consent to participate}

Signed informed consent was obtained from the patient for the publication of this case report. The patient granted consent for the images to be published.

\section{Author details}

'Premedical Science, College of Medicine, Chosun University, Gwangju, Republic of Korea. ${ }^{2}$ Department of Pathology, School of Medicine, Chosun University, Gwangju, Republic of Korea. ${ }^{3}$ Department of Internal Medicine, School of Medicine, Chosun University, Gwangju, Republic of Korea. ${ }^{4}$ Department of Neurosurgery, School of Medicine, Chosun University, Gwangju, Republic of Korea. ${ }^{5}$ Department of Laboratory Medicine, School of Medicine, Chosun University, Gwangju, Republic of Korea. ${ }^{6}$ Department of Laboratory Medicine, Chonnam National University Medical School, Gwangju, Republic of Korea.

\section{Received: 13 August 2016 Accepted: 8 December 2016}

\section{Published online: 14 January 2017}

\section{References}

1. Kershaw P, Freeman R, Templeton D, DeGirolami PC, DeGirolami U, Tarsy D, et al. Pseudallescheria boydii infection of the central nervous system. Arch Neurol. 1990;47:468-72. doi:10.1001/archneur.1990.00530040126029.

2. Husain S, Alexander BD, Munoz P, Avery RK, Houston S, Pruett T, et al. Opportunistic mycelial fungal infections in organ transplant recipients: emerging importance of non-aspergillus mycelial fungi. Clin Infect Dis. 2003; 37:221-9. doi:10.1086/375822

3. Husain S, Muñoz P, Forrest G, Alexander BD, Somani J, Brennan K, et al. Infections due to Scedosporium apiospermum and Scedosporium prolificans in transplant recipients: clinical characteristics and impact of antifungal agent therapy on outcome. Clin Infect Dis. 2005;40:89-99. doi:10.1086/426445.

4. Castiglioni B, Sutton DA, Rinaldi MG, Fung J, Kusne S. Pseudallescheria boydii (anamorph Scedosporium apiospermum). Infection in solid organ transplant recipients in a tertiary medical center and review of the literature. Medicine. 2002;81:333-48.

5. Kim MN, Shin JH, Sung H, Lee K, Kim EC, Ryoo N, et al. Candida haemulonii and closely related species at 5 university hospitals in Korea: identification, antifungal susceptibility, and clinical features. Clin Infect Dis. 2009;48:e57-61.

6. Clinical and Laboratory Standards Institute. Reference method for broth dilution antifungal susceptibility testing of filamentous fungi. 2nd ed, M38-A2. Wayne, PA: Clinical and Laboratory Standards Institute, 2008.

7. Lamaris GA, Chamilos G, Lewis RE, Safdar A, Raad II, Kontoyiannis DP. Scedosporium infection in a tertiary care cancer center: a review of 25 cases from 1989-2006. Clin Infect Dis. 2006;43:1580-4. doi:10.1086/509579.

8. Gallelli B, Viviani M, Nebuloni M, Marzano AV, Pozzi C, Messa P, et al. Skin infection due to alternaria species in kidney allograft recipients: report of a new case and review of the literature. J Nephrol. 2006;19:668-72.

9. Hsu CC, Chang SS, Lee PC, Chao SC. Cutaneous alternariosis in a renal transplant recipient: a case report and literature review. Asian J Surg. 2015;38:47-57. doi:10.1016/j.asjsur.2012.08.010

10. Pastor FJ, Guarro J. Alternaria infections: laboratory diagnosis and relevant clinical features. Clin Microbiol Infect. 2008;14:734-46. doi:10.1111/j.14690691.2008.02024.X.

11. Ruinemans GMF, Haagsma CJ, Hendrix R. Tenosynovitis caused by a pseudallescheria boydii infection and symptoms of reflex sympathetic dystrophy after a dog bite. J Clin Rheumatol. 2011;17:363-4. doi:10.1097/ RHU.0b013e31823279dd.

12. Ko JH, Hwang YJ, Kim JY, Lee YW, Choe YB, Ahn KJ. A case of concomitant tinea infection diagnosed with molecular biologic technique. Korean J Med Mycol. 2010;15:150-5.

13. Sangoi AR, Rogers WM, Longacre TA, Montoya JG, Baron EJ, Banaei N. Challenges and pitfalls of morphologic identification of fungal infections in histologic and cytologic specimens : A ten-year retrospective review at a single institution. Am J Clin Pathol. 2009;131:364-75. doi:10.1309/AJCP99000ZSNISCZ.

14. Uenotsuchi T, Moroi Y, Urabe K, Tsuji G, Koga T, Matsuda T, et al. Cutaneous Scedosporium apiospermum infection in an immunocompromised patient and a review of the literature. Acta Derm Venereol. 2005;85:156-9. doi:10. 1080/00015550410024553.

15. Ruxin TA, Steck WD, Helm TN, Bergfeld WF, Bolwell BJ. Pseudallescheria boydii in an immunocompromised host. Successful treatment with debridement and itraconazole. Arch Dermatol. 1996;132:382-4. doi:10.1001/ archderm.132.4.382

16. Gottesman-Yekutieli T, Shwartz O, Edelman A, Hendel D, Dan M. Pseudallescheria boydii infection of a prosthetic hip joint-an uncommon infection in a rare location. Am J Med Sci. 2011;342:250-3. doi:10.1097/MAJ. ob013e31821f9691.

17. Lackner M, de Hoog GS, Verweij PE, Najafzadeh MJ, Curfs-Breuker I, Klaassen $\mathrm{CH}$, et al. Species-specific antifungal susceptibility patterns of Scedosporium and Pseudallescheria species. Antimicrob Agents Chemother. 2012;56:2635-42.

18. Revankar SG, Sutton DA. Melanized fungi in human disease. Clin Microbiol Rev. 2010;23:884-928. doi:10.1128/CMR.00019-10.

\section{Submit your next manuscript to BioMed Central and we will help you at every step:}

- We accept pre-submission inquiries

- Our selector tool helps you to find the most relevant journal

- We provide round the clock customer support

- Convenient online submission

- Thorough peer review

- Inclusion in PubMed and all major indexing services

- Maximum visibility for your research

Submit your manuscript at www.biomedcentral.com/submit
) Biomed Central 